DOI: 10.30612/tangram.v4i4.11077

\title{
O reforço escolar como um espaço para superação de dificuldades em matemática
}

School reinforcement as a space to overcome difficulties in mathematics

El refuerzo escolar como espacio para superar las dificultades matemáticas

Ana Lucia do Carmo Narciso

Universidade Federal de Juiz de Fora (UFJF)

Carangola, Minas Gerais, Brasil

E-mail: narcisoaana@gmail.com

Orcid: 0000-0002-0336-3574

Adrielle Lourenço de Sá Universidade Candido Mendes (UCAM)

Porciúncula, Rio de Janeiro, Brasil

E-mail: adriellelourenconunes@gmail.com

Orcid: 0000-0002-1090-1289

Herman Fialho Fumiã

Universidade Federal de Viçosa (UFV)

Divino, Minas Gerais, Brasil

E-mail: adriellelourenconunes@gmail.com

Orcid: 0000-0002-1090-1289

Luciane da Silva Oliveira Universidade do Estado de Minas Gerais (UEMG) Espera Feliz, Minas Gerais,Brasil

E-mail: luciane.oliveira@uemg.br

Orcid: 0000-0001-6472-8824 
Resumo: A formação de professores caracteriza-se como o pilar de todas as reflexões feitas no âmbito educacional. Nesse sentido, ela assume um papel de centralidade na Educação, evidenciando a importância de que esse processo formativo estimule no licenciando o desenvolvimento de competências didáticas que colaborem para a formação de sua identidade profissional e, nesse contexto, as atividades de extensão passam a representar uma contribuição enriquecedora à formação inicial, por proporcionar momentos de vinculação da teoria à prática. Neste trabalho relatamos as experiências vivenciadas durante a participação em um projeto de extensão desenvolvido em uma escola da rede pública Estadual de ensino, situada em Carangola, Minas Gerais, cujo objetivo era oferecer aulas de reforço de matemática aos alunos que apresentavam baixo rendimento nesta disciplina. Constatamos que houve um progresso significativo na aprendizagem dos alunos participantes e que a experiência proporcionada pelo projeto foi enriquecedora para todos os atores envolvidos no processo.

Palavras chave: Reforço escolar. Matemática. Relato de experiência.

Abstract: Teacher education is characterized as the pillar of all reflections made in the educational sphere. In this sense, it assumes a central role in Education, highlighting the importance that this training process encourages the development of teaching skills that contribute to the formation of their professional identity in the licensing process and, in this context, extension activities come to represent a enriching contribution to initial training, by providing moments of linking theory to practice. In this paper we report the experiences lived while participating in an extension project developed in a public school in the state education system, located in Carangola, Minas Gerais, whose objective was to offer math reinforcement classes to students who had low performance in this subject. We found that there was significant progress in the learning of participating students and that the experience provided by the project was enriching for all actors involved in the process.

Keywords: School reinforcement. Mathematics. experience report.

Resumen: La formación del profesorado se caracteriza por ser el pilar de todas las reflexiones realizadas en el ámbito educativo. En este sentido, asume un rol central en Educación, resaltando la importancia que este proceso de formación incentiva el desarrollo de habilidades docentes que contribuyan a la formación de su identidad profesional en el proceso de licenciamiento y, en este contexto, las actividades de extensión pasan a representar una contribución enriquecedora a la formación inicial, al brindar momentos de vinculación de la teoría con la práctica. En este trabajo se reportan las experiencias vividas al participar en un proyecto de extensión desarrollado en una escuela pública del sistema educativo estatal, ubicada en Carangola, Minas Gerais, cuyo objetivo fue ofrecer clases de refuerzo matemático a estudiantes que tenían bajo rendimiento en esta asignatura. Encontramos que hubo un avance significativo en el aprendizaje de los estudiantes participantes y que la experiencia brindada por el proyecto fue enriquecedora para todos los actores involucrados en el proceso.

Palabras clave: Refuerzo escolar. Matemáticas. Informe de experiencia.

Recebido em

$21 / 02 / 2020$

Aceito em

14/08/2021 


\section{CONSIDERAÇÕES INICIAIS}

A formação de professores caracteriza-se como o pilar de todas as reflexões feitas no âmbito educacional, principalmente quando se parte do pressuposto de que repousa sobre a educação a responsabilidade de atuar na formação de cidadãos aptos a tomarem criticamente, decisões cujo impacto poderá ser observado nos mais diversos setores da sociedade.

Nesse contexto a formação do profissional docente assume um papel de centralidade no meio educacional, evidenciando a importância de que esse processo formativo busque, através dos mais variados modelos e práticas de ensino, estimular no licenciando no desenvolvimento de competências didáticas que colaborem para formação de sua identidade profissional, surtindo efeitos principalmente na aptidão para selecionar metodologias de ensino que tornem seu processo de transposição didática mais eficaz.

Fomentar oportunidades de que o licenciando possa vivenciar experiências de vinculação da teoria à prática, através de sua aproximação com sua futura área de atuação torna-se um componente fundamental no processo de constituição da identidade pedagógica de cada profissional, e nesse sentido, as atividades de extensão passam a representar uma contribuição enriquecedora à formação inicial.

De acordo com o "Programa institucional de apoio à extensão da universidade do estado de Minas Gerais/UEMG", o conceito de Extensão Universitária se relaciona com o posicionamento da Universidade frente à sociedade na qual esta se estabelece. Apontando assim, para uma aproximação da comunidade acadêmica com a sociedade através de um trabalho de pesquisa básica e prática, cujo intuito é (re) significar o processo de ensino-aprendizagem através de intervenções no contexto em que essas atividades se desenvolvem.

As atividades extensionistas proporcionam um intercâmbio de experiências entre licenciandos e professores de diversas áreas do conhecimento, de maneira a 
contribuir para a formação acadêmica e profissional dos estudantes de graduação (UEMG, 2015). Em outras palavras,

a extensão universitária é entendida como processo interdisciplinar, educativo, cultural e científico que, articulada ao Ensino e à Pesquisa, difunde o conhecimento produzido na universidade e abre espaço para a comunidade participar da vida acadêmica (...) Essa movimentação torna favorável a abertura de diálogos e afirma a extensão como uma via de mão dupla na qual professores e estudantes encontram, na sociedade, a oportunidade de elaborar a práxis de um conhecimento acadêmico (UEMG, 2019).

O presente artigo teve como objetivo relatar as experiências vivenciadas por duas discentes do curso de Licenciatura em Matemática, durante a participação em um projeto de extensão no ano de 2017, em uma escola da rede pública estadual de ensino localizada em Carangola, Minas Gerais.

O projeto em questão visou oferecer apoio pedagógico às escolas da rede pública de ensino, através de aulas de reforço escolar ministradas por alunos voluntários do curso de licenciatura em Matemática da Universidade do Estado de Minas Gerais (UEMG) - Unidade Carangola. Desse modo, ao participar dessa atividade extensionista o licenciando teve a oportunidade de aprimorar e refletir sobre suas práticas pedagógicas através do contato com o ambiente escolar.

A necessidade de se implementar ações similares as desenvolvidas pelo projeto se torna mais evidente quando se observa os baixos índices de proficiência em Matemática, verificados em avaliações externas como o Simave (Sistema Mineiro de Avaliação da Educação Pública), a nível estadual, o Saeb (Sistema de Avaliação da Educação Básica), a nível nacional e o Pisa (Programa Internacional de Avaliação de Estudantes), a nível internacional.

Os dados do Saeb de 2017 revelam que apenas $4,52 \%$ dos estudantes que cursam o Ensino Médio alcançaram os índices de aprendizagem considerados adequados pelo Ministério da Educação (MEC). Para os anos iniciais do Ensino Fundamental o percentual obtido foi de $15,5 \%$, também foi verificado que somente $4,5 \%$ dos discentes que cursam os anos finais do Ensino Fundamental obtiveram os resultados esperados pelo MEC (INEP, 2017). 
Os baixos índices de aproveitamento na disciplina de Matemática também podem ser observados através dos dados obtidos com as avaliações do Pisa, aplicadas no ano de 2015. Esses resultados mostram que 70,3\% dos estudantes encontram-se abaixo nível considerado necessário para que os jovens estejam aptos ao exercício pleno da cidadania, de acordo com a OCDE (Organização para a Cooperação e Desenvolvimento Econômico) (Brasil, 2016).

Diversos aspectos podem ser condicionantes para que haja uma aprendizagem significativa em Matemática. Dentre eles podemos citar a escolha de metodologias de ensino que respeitem as características individuais e os diferentes ritmos de assimilação de cada aluno, para que sejam criados ambientes de aprendizagem favoráveis ao desenvolvimento dos alunos.

Sabendo que durante as aulas regulares dificilmente o professor consegue atender a todos os alunos de maneira mais individualizada devido ao número de alunos por turma, as aulas de reforço escolar surgem como uma alternativa capaz de atuar na consolidação das aprendizagens de maneira efetiva, ao proporcionar um atendimento mais direcionado a cada uma das dificuldades apresentadas no entendimento da Matemática.

\section{O PROJETO dE EXTENSÃO “AMIgOS dA MATEMÁTICA"}

O projeto de extensão "Amigos da Matemática" foi desenvolvido pelo curso de Matemática e o Núcleo de Pesquisa e Extensão (NUPEX) da Universidade do Estado de Minas Gerais (UEMG) - Unidade Carangola, e teve como objetivo geral, destinar atividades de reforço escolar às instituições da rede pública estadual e municipal de ensino dos municípios situados nas mediações da Unidade.

Dessa maneira, ao oferecer aulas de reforço na disciplina de Matemática para alunos do Ensino Fundamental e Médio, o projeto promoveu a aproximação da comunidade acadêmica às escolas de Educação Básica. Além disso, tal atividade extensionista contribui para que o licenciando estivesse inserido no contexto escolar, proporcionando aprimoramento e reflexão de suas próprias metodologias de ensino. 
Dentre os objetivos específicos do projeto encontram - se: o estímulo ao desenvolvimento de aspectos inerentes ao pensamento lógico matemático e à autoconfiança no processo de aprendizagem, através da superação de eventuais bloqueios relacionados à compreensão dos conteúdos abordados; destacar a importância de uma aprendizagem cooperativa, pautada na socialização de dúvidas e aprendizados; propor atividades que lancem mão do aspecto lúdico como meio para superar dificuldades de assimilação da matéria lecionada e contribuir para uma melhora no desempenho da escola em avaliações externas, através do auxílio em processos de interpretação e resolução de situações - problema.

Tendo em vista que muitas vezes o grande contingente de alunos nas turmas impossibilita que o professor destine uma atenção mais individualizada a alunos que apresentam problemas de aprendizagem em Matemática, a ocorrência do projeto se justificou por ser um instrumento de auxílio didático a professores e alunos que vivem essa realidade. Com efeito, as atividades de reforço escolar ofereceram ao alunado a oportunidade de superar eventuais dificuldades relacionadas ao aprendizado da Matemática por proporcionar um ambiente em que a aprendizagem ocorresse de maneira contextualizada e que respeitasse o ritmo de assimilação de cada discente.

\section{DIRETRIZES DO PROJETO}

As atividades do projeto foram desenvolvidas em uma escola da rede pública estadual de ensino, situada no município de Carangola/MG. Essa instituição oferece vagas para o Ensino Fundamental II, Ensino Médio e Educação de Jovens e Adultos, e concentra atividades letivas nos turnos matutino, vespertino e noturno.

Nossas atividades ocorreram entre os meses de maio e dezembro do ano de 2017. Em um primeiro momento foi realizada uma reunião que contou com a participação da equipe de coordenação do projeto e a equipe pedagógica da escola a fim de traçar as diretrizes de funcionamento das atividades, bem como determinar os horários e 0 local onde essas aconteceriam. 


\section{Universidade Federal da Grande Dourados}

Após esse encontro, ficou determinado que as aulas de reforço seriam destinadas às turmas do $6^{\circ}$ ano do Ensino Fundamental, dos turnos matutino e vespertino, as quintas e sextas-feiras e em horários diferentes das aulas regulares dos alunos. Além disso, ficou acordado que a escola forneceria todo o material necessário ao atendimento dos alunos, como por exemplo, impressões das atividades, jogos educacionais destinados ao ensino da matemática, lápis, borracha, papel para escrita e um arquivo para que guardássemos as atividades aplicadas e assim fosse possível acompanhar o progresso dos alunos.

Em seguida, a responsável por nos oferecer auxílio pedagógico durante a realização do projeto, nos forneceu os dados de uma avaliação diagnóstica aplicada pela direção da escola. O objetivo dessa atividade era identificar em quais conteúdos os alunos apresentavam maior defasagem e quais desses estavam aquém do nível de conhecimento esperado para o ciclo de escolaridade em que se encontrava.

A respeito das avaliações diagnósticas, Cortesão (2002, p. 39) destaca que seu objetivo é: "a) identificar as competências dos alunos no início de uma fase de trabalho" ou "b) colocar o aluno num grupo ou num nível de aprendizagem e/ou prever o que muito provavelmente virá a ocorrer na sequência das situações educativas desenvolvidas". A autora destaca ainda, que essa maneira de avaliar oferece ao professor elementos importantes para que seja adotada uma estratégia de ensino coerente com os conhecimentos e características de cada aluno.

Foram selecionados 44 alunos, que obtiveram resultado abaixo do esperado na avaliação diagnóstica para participar das aulas de reforço. É importante destacar que entre esses alunos também se encontravam aqueles que foram selecionados após indicação das professoras de Matemática. Todavia, apesar desse número de alunos que apresentavam algum déficit em relação ao aprendizado da Matemática, apenas 19 compareceram continuamente desde no início das atividades do projeto.

Nossa equipe era composta por quatro licenciandos do curso de Matemática da UEMG - Unidade Carangola, e a fim de tornar nosso auxílio pedagógico mais eficaz, optamos, juntamente com a coordenadora do projeto, por organizar a turma em 
grupos, de acordo com as dificuldades demonstradas por eles na avaliação diagnóstica.

Assim, durante as aulas, organizávamos a turma em duplas ou trios de alunos que apresentavam a mesma defasagem em geometria, interpretação de problemas, frações ou operações básicas, por exemplo, para que pudéssemos dar uma orientação mais direcionada a cada tipo de dificuldade.

Cunha (2016), afirma em sua tese que o reforço escolar cria um ambiente de aprendizagem favorável à implementação de técnicas de ensino diversificadas, por ser um momento onde os alunos têm a oportunidade de rever conteúdos que não foram compreendidos no horário regular de aulas, sobretudo por oferecer um acompanhamento mais individualizado, devido à pequena quantidade de alunos que normalmente participam das aulas.

Para esse autor, a necessidade de atuar na aprendizagem dos alunos de maneira significativa tem se revelado uma premência no meio educacional, evidenciando a importância de que as aulas se deem em contextos enriquecedores e contextualizados de experiências cotidianas, sendo que o reforço escolar pode ser considerado um momento propício à consolidação dessas aprendizagens.

Com o intuito de criar um ambiente favorável a ocorrência de aprendizagens significativas, tínhamos encontros semanais com a orientadora do projeto, onde elaborávamos as atividades e discutíamos como os conteúdos seriam ministrados. Assim, buscávamos desenvolver diferentes técnicas de ensino que fossem capazes de respeitar as dificuldades individuais e os diferentes ritmos de assimilação característicos de cada aluno.

\section{IMPLEMENTAÇÃO DO PROJETO}

Para que pudéssemos traçar um plano de ação, fizemos em um primeiro momento a correção da avaliação diagnóstica, a fim de detectar quais eram as principais dificuldades apresentadas pelos alunos. Mediante esses resultados, constatamos que os principais erros se concentravam nas questões que envolviam frações, interpretação e resolução de situações-problema, interpretação de gráficos, 


\section{Universidade Federal da Grande Dourados}

radiciação, potenciação, expressões numéricas e cálculos envolvendo as quatro operações básicas.

Logo após, como um primeiro ato de intervenção pedagógica, nos propusemos a refazer com os alunos as questões da avaliação aplicada pela escola com a intenção de entender, tendo como base os relatos dos próprios discentes, quais eram os principais problemas encontrados por eles ao resolver a atividade.

Através desses relatos, pudemos perceber que as dificuldades no entendimento das operações básicas era uma unanimidade entre eles, sendo que a grande maioria tinha problemas ou não sabia como resolver a operação de divisão.

Também verificamos que muitos alunos apresentavam uma grande ansiedade com relação ao aprendizado da Matemática por apresentarem dificuldades em assimilar os algoritmos da divisão e da multiplicação, ou por terem dificuldades em reconhecer o procedimento necessário à resolução de algumas situações - problema, por exemplo.

Todavia, observamos que, apesar de terem dificuldades para seguir uma lógica de resolução dotada de um rigor matemático conforme foram ensinados em sala de aula, muitos alunos conseguiam chegar ao resultado esperado, elaborando procedimentos de resolução bastante idiossincráticos que obedeciam a uma lógica matemática coesa.

Essa observação nos remeteu a importância de estimular aprendizagens autônomas e dinâmicas através de um processo de transposição didática que dê liberdade para que os alunos compreendam que, a aprendizagem da Matemática não se reduz à assimilação e aplicação de regras e algoritmos. Isso posto, destaca-se a importância de se colocar o aluno como sujeito de seu conhecimento, conferindo a ele a oportunidade de assumir um papel ativo na consolidação de suas aprendizagens.

Tendo como base esse pressuposto, chegamos à conclusão de que os jogos educacionais poderiam representar uma maneira de criar ambientes de aprendizagem eficazes, e resolvemos utilizar esse recurso didático em nossas aulas sempre que possível. 
Sobre a utilização de jogos educacionais como instrumentos de mediação pedagógica, Silva e Kodama (2004) e Lara (2004) destacam que, esse tipo de metodologia é capaz de estimular a autoconfiança dos alunos e os envolver na resolução dos problemas que vão surgindo no decorrer da atividade, levando a aprendizagens ativas, dinâmicas e cooperativas. Além disso, os autores enfatizam que a utilização desses objetos de aprendizagem pode auxiliar no aprimoramento da habilidade de socializar os aprendizados e de raciocinar logicamente.

O espaço para arquivo concedido pela escola nos permitiu fazer um mapeamento de todas as atividades aplicadas, e com isso constatamos que houve um avanço na aprendizagem dos alunos aos quais acompanhamos durante o desenvolvimento do projeto. Percebemos também que, a cada encontro a resistência quanto à aprendizagem da Matemática foi diminuindo gradativamente e abrindo espaço para a superação de dificuldades por meio da retomada dos conteúdos ministrados nas aulas regulares.

Esse avanço dos alunos também pôde ser observado pelas professoras regentes de turma e pela direção da escola, que mostraram grande contentamento com a evolução dos discentes. Em algumas conversas com a equipe pedagógica da instituição, foi ressaltada a importância de iniciativas como a do projeto para a redução da evasão escolar e do fracasso na disciplina de Matemática.

\section{Universidade Federal da Grande Dourados}

\section{CONSIDERAÇÕES FINAIS}

O número excessivo de alunos por turma pode inviabilizar um processo de ensinoaprendizagem efetivo que alcance todos os discentes de forma satisfatória, posto que impossibilita que o professor dê uma atenção mais individualizada àqueles que possuem maiores dificuldades em relação à aprendizagem de Matemática. 


\section{Universidade Federal da Grande Dourados}

Desta maneira, as atividades de reforço escolar se tornam importantes instrumentos de auxílio pedagógico por oferecerem ao alunado a oportunidade de avançar em suas aprendizagens através de metodologias de ensino diferenciadas que respeitam as características e os estilos de aprendizagem de cada aluno.

Durante a realização do projeto "Amigos da Matemática" foram utilizadas metodologias de ensino que enfatizaram o papel do estudante como sujeito do processo de ensino - aprendizagem, abrindo espaço para a ocorrência de aprendizagens cooperativas e dinâmicas. Destacamos ainda, que as atividades propostas apresentavam os conteúdos de maneira lúdica e criativa, estimulando o interesse e o desenvolvimento dos discentes.

Os resultados positivos alcançados com as aulas de reforço nos permitem afirmar que essas técnicas de ensino foram bem aceitas pelos alunos, fazendo com que a ansiedade em relação à aprendizagem da Matemática fosse reduzida.

No que diz respeito às impressões dos voluntários do projeto, percebemos através das trocas de experiências que a participação no projeto possibilitou a reflexão de nossas práticas de ensino, através da convivência antecipada com situações que enfrentaremos em nosso exercício profissional. De modo geral, destacamos que a participação no projeto foi uma experiência enriquecedora a todos os envolvidos, em função dos resultados observados. 


\section{Universidade Federal da Grande Dourados}

\section{REFERÊNCIAS}

Brasil. (2016). Brasil no PISA 2015: Análises e reflexões sobre o desempenho dos estudantes brasileiros. São Paulo: Fundação Santillana.

Cortesão, L. (2002). Formas de ensinar, formas de avaliar: Breve análise de práticas correntes de avaliação. In: P. Abrantes, \& F. Araújo (Coord.), Reorganização curricular do ensino básico: Avaliação das aprendizagens das concepções às práticas (pp. 3742). Lisboa: Ministério da Educação, Departamento da Educação Básica.

Cunha, E. C. (2016). Reforço Escolar: O uso de jogos e materiais manipuláveis no ensino de frações. (Dissertação de mestrado). Universidade Federal de RondôniaUNIR, Porto Velho, RO, Brasil.

Instituto Nacional de Estudos e Pesquisas Educacionais Anísio Teixeira. (2017). Sistema de Avaliação da Educação básica. Recuperado de http://portal.mec.gov.br/index.php?option=com docman\&view=download\&alias=9416 1-saeb-2017-versao-ministro-revfinal\&category slug=agosto-2018 pdf\&ltemid=30192.

Lara, I. C. M. (2004). Jogando com a Matemática de $5^{\underline{a}}$ a $8^{\underline{a}}$ série. São Paulo: Editora Rêspel.

Silva, A. F., \& Kodama, H. M. Y. (2004, outubro). Jogos no ensino da matemática. Anais da II Bienal da Sociedade Brasileira de Matemática, Salvador, BA, Brasil.

Universidade do Estado de Minas Gerais. (2015). Programa institucional de apoio à extensão da universidade do estado de Minas Gerais/UEMG. Belo Horizonte: Autor. Recuperado de http://www.2018.uemg.br/downloads/extensao/Programa_Institucional_Apoio_Extens ao_2015.pdf

Universidade do Estado de Minas Gerais. (2019). Extensão. Belo Horizonte: Autor. Recuperado de https://www.uemg.br/extensao 